\title{
Alcohol and Drug Use at the U.S.-Mexico Border - Does Cross-Border Mobility make a Difference?
}

Received: January 06, 2016; Accepted: January 14, 2016; Published: January 21, 2016

\section{Introduction}

People of Mexican origin constitute the largest subgroup of Hispanics in the U.S., comprising over $65 \%$ of this group and $11 \%$ of the total U.S. population, a figure which is expected to double by 2050 [1]. Mexicans represent 29\% of the U.S. immigrant population, with Mexico being the largest contemporary source of immigration. The U.S. side of the border has become home to many of these individuals, as well as a staging area for migration to the interior of the U.S. [2]. This pattern of immigration suggests an extremely high potential for alcohol and drug use patterns and problems in Mexico and at the U.S. border to spread and become generalized outside of the border region, making this region especially significant to public health in the U.S. as a whole.

Among all U.S. Hispanics, $54 \%$ live in the four border states, which stretch approximately 2,000 miles (from the Pacific Ocean to the Gulf of Mexico), and include 25 border counties. The U.S. border region is a highly disadvantaged area, characterized by high rates of poverty with low rates of education and employment, and is economically interdependent with Mexico. The major metropolitan areas on both sides of the border (sister cities) are important points of commerce, population growth, and heightened trans-border movement. Border residents on both sides have typically been able to enter a designated zone with fewer legal restrictions than those which apply to secondary checkpoints further away from the border. This facilitated movement back and forth across the border, allowing individuals to shop, visit, and conduct business or work, as well as to obtain medical services and pharmaceuticals. The enhanced access to pharmaceutical drugs in Mexico (many of which are not available in the U.S.), enabled cheap and easy access to a variety of drugs for recreational purposes. Known as "drug tourism", this has historically been a major reason for U.S. residents to cross the border to Mexico. The U.S.-Mexico border is unlike other border regions, for example the U.S.-Canadian border, in relation to the large volume of those crossing frequently, the vast majority of whom are residents of the border area (on both its sides) and coming primarily from the municipalities next to the crossing itself.

\section{Substance Use at the Border}

Recent media attention directed at elevated drug trafficking and drug-related violence at the border, coupled with increasing policy and legal tension related to heightened security measures

\section{Cheryl J Cherpitel \\ Alcohol Research Group, 6475 Christie Avenue, Suite 400, Emeryville, CA 94608, USA}

Corresponding author: Cheryl J Cherpitel

” ccherpitel@arg.org

Alcohol Research Group, 6475 Christie Avenue, Suite 400, Emeryville, CA 94608, USA.

\section{Tel: 5108985843}

Fax: 510 985-6459

Citation: Cherpitel CJ. Alcohol and Drug Use at the U.S.-Mexico Border - Does CrossBorder Mobility make a Difference?. J Drug Abuse. 2016, 2:1.

at the border, may have affected cross-border fluidity, however. While there is reason to believe that such stressors may also affect substance use patterns and problems at the border, little has been known regarding the impact of these issues on individuals living on either side. Prior research has suggested that Mexicanorigin men may be at higher risk for alcohol-related problems than others, and those living along the U.S. border may be at even higher risk, especially among those younger [3], but little epidemiologic evidence has existed to support this. Especially concerning in this regard is the potential vulnerability of younger people to excessive drinking and related problems, due to greater availability of alcohol and at lower cost, and under-enforced legal drinking age in Mexican border cities. Mexican bars cater to these individuals, facilitating heavy drinking by advertising inexpensive alcohol in large quantities. Public drunkenness is also accepted in bars and near border crossings, where the volume of foot and vehicle traffic prohibits citations for public drunkenness, underage drinking, or drinking and driving [4]. Studies of those crossing the border between midnight and 4 a.m. on weekend nights found two-thirds to three-quarters were Mexican-American pedestrians returning from a bar or restaurant, with half reporting the intention to get drunk and a third with a blood alcohol concentrations (BAC) of 0.08 or greater [5]. One study conducted in 2002-03 found that while volume of consumption among Mexican-Americans living at the border was no greater than that for those living off the border, alcohol abuse and dependence were higher, with $23 \%$ reporting 
binge drinking in the last month and $12 \%$ reporting symptoms of alcohol dependence, which had doubled over the previous six years [6]. This same study also found life-time and past-year drug use and problems increased significantly, paralleling the rise in alcohol use and problems during the same time period.

\section{The U.S.-Mexico Study of Alcohol and Related Conditions (UMSARC)}

Given this prior research and characteristics of border life, including high mobility of the population on both sides, a study was undertaken to examine whether the border is a risk environment for alcohol and drug problems, and the role of border proximity and variables related to crossing the border (cross-border mobility) on substance use and problems.

This study, called the U.S.-Mexico Study of Alcohol and Related Conditions (UMSARC) is one of two recent epidemiological studies (the second conducted by Caetano [3]) of the border region addressing alcohol use, and the only known study to incorporate parallel data collection, simultaneously, on both sides of the border. In the U.S., data were collected on 1565 Mexican-origin respondents, aged 18-65, living in three border "sister cities" located in Texas and 771 respondents living in the non-border city of San Antonio, using area probability sampling techniques with face-to-face household interviews. "Sister Cities" are those which span the border on both sides, facilitating cross-border mobility in both directions. Texas includes almost two-thirds of all U.S. border counties, and the single-state focus limited potential heterogeneity in geographic, cultural and sociopolitical factors in studying border effects on substance use. As expected, the UMSARC study found the prevalence of alcohol use disorders (AUD) was greater among those living at the border, compared to the interior site of the study, at the same average monthly volume of alcohol consumption and the same number of heavy drinking days during the last year [7], with co-occurring hazardous alcohol and drug use also more common among those living at the border compared to the interior [8].

\section{Cross-Border Mobility}

Since prior research had suggested that individuals crossing the border to Mexico may be especially vulnerable to substance use and related problems, the UMSARC study hypothesized that frequency of crossing the border, length of stay, and reasons for crossing (especially those related to obtaining pharmaceuticals and for nightlife/drinking) would be positively predictive of heavy drinking, AUD, and co-occurring heavy drinking and drug use. While cross-border mobility was predictive of substance use, it was most predictive among those aged 18-29, with reasons for crossing (for pharmaceuticals or for nightlife/drinking) predictive of all three substance use variables [9]. Greater frequency of crossing and longer visits were also predictive of AUD, while greater frequency of crossing was predictive of co-occurrence of heavy drinking and drug use. Among those older, only longer visits and crossing for pharmaceutical reasons were predictive of both heavy drinking and co-occurrence of heavy drinking and drug use.

Surprisingly, the number of those who reported crossing the border in the last year was not as high as expected, with only $40 \%$ crossing during this time. However, the majority who did cross, crossed on more than two occasions, but typically stayed less than a day. The number of those crossing for either pharmaceutical reasons or for nightlife/drinking was also much smaller than expected (10\%).

\section{Discussion}

While "drug tourism" and attending bars or nightclubs have been considered to be major reasons for crossing the border to Mexico, the UMSARC is the first epidemiological study documenting reasons for crossing and related variables, and the proportion of those crossing who crossed for either of these reasons was considerably lower than expected. One explanation for this finding is the fact that only the main reason for crossing the border was obtained, and many of those who reported crossing primarily to visit family and friends (over half of those who reported crossing) may also have obtained pharmaceuticals on these trips, or have gone to bars and nightclubs with their family and friends. Future research in this area should consider all reasons for crossing the border, with associated frequency and length of stay.

The likelihood of crossing the border may also be affected by the length of time an individual has lived on the U.S. side of the border, with more recent immigrants crossing to Mexico on a more regular basis. Nativity and recency of immigration were not examined in this context, however. Certainly different age groups are likely to cross the border for different reasons, and prior research has shown underage individuals are especially likely to cross in order to avail themselves of inexpensive alcohol. While the UMSARC did not include sufficient numbers of those under 21 to examine this, such a supposition may be partially reflected in the findings pertaining to those aged 18-29, among whom crossing for drinking/nightlife was highly predictive of substance use. Although reasons for crossing the border may be more important than frequency of crossing or time spent on a visit in relation to substance use and problems, exposure related to frequency of crossing and time spent in Mexico also appear to be important, especially for those over 30 , and is an area deserving more attention in examining the effects of cross-border mobility on substance use and problems.

Reasons for crossing the border to Mexico may have important public health implications spanning both sides. UMSARC findings highlight the importance of "drug tourism" in substance use across the age spectrum, which is highly important for informing intervention and prevention strategies within the border context. Crossing for pharmaceutical reasons was the strongest predictor of co-occurring heavy drinking and drug use across all ages in the UMSARC sample, suggesting the importance of substance use treatment aimed not only at alcohol, but at co-morbid alcohol and drug use as well, in this border population. Cheap and easy access to alcohol creates a situation where thousands of young people cross the border to drink, but the sheer number of these individuals prohibits citations for drinking and driving [4]. Implementation of a 2 a.m. bar closing policy in a Mexican border city, replacing a 5 a.m. closing, was followed by a reduction in positive BAC [10], and suggests the efficacy of environmental control strategies in limiting alcohol availability. 
Data collected at the U.S. - Mexico border may have important applicability to border contexts in other countries, as well; for example, in adjacent countries in which alcohol/and or drug pricing and availability varies greatly. Data such as the UMSARC can potentially identify key problem areas and risk factors related to alcohol and drug use at the border for informing intervention and prevention strategies within the border context. It is important to note, however, that the UMSARC data were crosssectional in nature, and do not allow us to determine whether individuals with alcohol and drug problems cross the border to avail themselves of an environment which facilitates their substance use, or whether alcohol and drug use and problems result from this environmental context. Future research is needed to elucidate the manner in which cross-border mobility may explain substance use and problems.

On a final note, the proportion of study respondents reporting crossing the border in the last year was considerably less than expected, suggesting that data from UMSARC may be considered new baseline data documenting the substantial decrease in the number of U.S. border residents traveling to Mexico, due to U.S. governmental policy restrictions limiting the former ease with which U.S. residents could cross the border to Mexico. 


\section{Acknowledgement}

Supported by a grant from the U.S. National Institute on Alcohol Abuse and Alcoholism (R01 AA01836540).

\section{References}

1 Passel JS, Cohn DV (2008) U.S. Population Projections: 2005-2050, PEW Research Center, Washington, DC, USA.

2 Koerber K (2007) Domestic Migration Flows for States from the 2005 American Community Survey Annual Meeting of the Population Association of America, New York City, USA.

3 Caetano R, Vaeth PAC, Mills BA, Rodriguez LA (2013) Alcohol abuse and dependence among U.S.-Mexico border and non-border Mexican Americans. Alcoholism: Clinical and Experimental Research 37: 847-853.

4 Lange JE, Voas RB (2000) Youth escaping limits on drinking: binging in Mexico. Addiction 95: 521-528.

5 Lange JE, Voas RB, Johnson MB (2002) South of the border: a legal haven for underage drinking. Addiction 97: 1195-1203.
6 Wallisch LS, Spence RT (2006) Alcohol and drug use, abuse, and dependence in urban areas and colonias ofthe Texas-Mexico border. Hispanic Journal of Behavioral Sciences 28: 286-307.

7 Cherpitel CJ, Ye Y, Bond J, Sarah EZ, Guilherme B, et al. (2015) Border effects on DSM-5 alcohol use disorders on both sides of the U.S.Mexico border. Drug and Alcohol Dependence 148: 172-179.

8 Borges G, Zemore SE, Orozco R, Cherpitel CJ, Ye Y, et al. (2015) Cooccurrence of alcohol, drug use, DSM-5 alcohol use disorder, and symptoms of drug use disorder on both sides of the U.S.-Mexico border. Alcoholism: Clinical and Experimental Research 39: 679-687.

9 Cherpitel CJ, Ye Y, Zemore SE, Bond J, Borges G (2015) The effect of cross-border mobility on alcohol and drug use among MexicanAmerican residents living at the U.S.-Mexico border. Addictive Behaviors 50: 28-33.

10 Voas RB, Lange JE, Johnson MB (2002) Reducing high-risk drinking by young Americans south of the border: the impact of a partial ban on sales of alcohol. Journal of Studies on Alcohol 63: 286-292. 